\title{
Prevalence of cognitive impairments following chemotherapy and its relationship to depression in Japanese breast cancer survivors: an exploratory
}

\section{study}

\begin{abstract}
Background: Mild to moderate cognitive impairments following adjuvant chemotherapy for breast cancer are reported in $15 \%-75 \%$ of breast cancer survivors (BCSs). However, research in this area is surprisingly limited compared to other psychological problems in Japanese population. This study aimed to elucidate the prevalence of chemotherapy-associated cognitive impairment and its relationship to depression in Japanese breast cancer survivors (BCSs).

Methods: Cross-sectional surveys were conducted online in a Japanese breast cancer advocacy group. The survey involved a clinical self-reported questionnaire for perceived cognitive impairments (PCIs) and the Hospital Anxiety and Depression Scale (HADS). The prevalence of PCIs and depression and the relationship between PCIs and depression were analyzed.

Result: A total of 173 survivors were enrolled in this study, of which 98 (56.6\%) were treated with chemotherapy. Of the survivors treated with chemotherapy, $43 \%$ were considered depressed. The prevalence of PCIs in the survivors treated with chemotherapy was $79 \%$, and $43 \%$ experienced more than three types of PCIs. However, the recognition rate of these PCIs by other people was significantly lower than the prevalence of PCIs in survivors treated with chemotherapy $(p<0.0001)$. Furthermore, multiple regression analysis revealed that the number of PCIs significantly correlated with the total HADS score in survivors treated with chemotherapy $(\beta=0.320, \mathrm{p}=0.001)$.

Conclusion: Our results suggest that PCIs following chemotherapy are not negligible in Japanese BCSs and could be important factors contributing to the development of depression.
\end{abstract}

Keywords: breast cancer survivors, cognitive impairment, depression, chemotherapy, chemobrain
Volume II Issue 2 - 2018

\author{
Hitoshi Tanimukai, ${ }^{1,2}$ Tomoko Matsui ${ }^{3}$ \\ 'Faculty of Human Health Sciences, Graduate School of \\ Medicine, Kyoto University, Japan \\ ${ }^{2}$ Department of Palliative Medicine, Kyoto University Hospital, \\ Japan \\ ${ }^{3}$ Graduate School of Human Sciences, Osaka University, Japan
}

\begin{abstract}
Correspondence: Hitoshi Tanimukai, Palliative Care Center, Department of Palliative Medicine, Kyoto University Hospital, 54 Kawaharacho, Syogoin, Sakyou-ku, Kyoto City, Kyoto, 6068507, Japan, Tel +8175 7514 629, Fax +8175 7514 423, Email
\end{abstract} tanimuki@kuhp.kyoto-u.ac.jp

Received: March 06, 2018 | Published: March 12, 2018

\section{Introduction}

Breast cancer is currently the most common malignancy in females world-wide. In Japan, an estimated 89,400 women were affected by breast cancer in 2015. Multiple randomized trials have shown that adjuvant chemotherapy improves long-term survival for most women with breast cancer. However, mild to moderate cognitive impairment following adjuvant chemotherapy for breast cancer, also known as "chemofog" or "chemobrain," are reported in $15 \%-75 \%$ of breast cancer survivors (BCSs). ${ }^{1-6}$ Several hypotheses regarding the etiology of chemotherapy-associated cognitive impairments have been proposed, including disruption of hippocampal cell proliferation and neurogenesis, ${ }^{7}$ chronic inflammation, ${ }^{8,9}$ increased oxidative stress, ${ }^{8}$ white matter disruption, ${ }^{10-12}$ and long-term changes in cerebral blood flow and metabolism. ${ }^{13}$ However, detailed mechanisms and interventions for chemotherapy-associated cognitive impairments have not been established.

Chemotherapy-associated cognitive impairment is defined as impairment in cancer survivors' memory, learning, attention/ concentration, reasoning, executive function, processing speed, and visuospatial skills during chemotherapy and after its discontinuation. ${ }^{14}$
In contrast with neurodegenerative dementias such as Alzheimer disease, relatively subtle impairment is found in neurocognitive tests, and the performance of cancer survivors treated with chemotherapy often falls within the average range, ${ }^{5}$ even though they experience difficulties caused by cognitive impairments. Given this situation, a variety of problems performing household chores or occupational activities that are associated with cognitive impairments may be missed or misunderstood by those close them, such as family members. This may result in emotional and/or behavioral effects and cause cancer survivors to feel isolated from other people and/or social activities, leading to depression. Thus, cognitive impairment is an important symptom in cancer survivors. However, research in this area is surprisingly limited compared to other psychological problems, such as depression. ${ }^{15}$

At present, an increasing number of studies are being conducted to investigate chemotherapy-associated cognitive impairment; however, these studies are primarily conducted in Western countries. Although researchers in Asian countries have gradually begun to focus on this condition, few studies have addressed this issue in Japanese cancer survivors. 
The aims of the present study were to investigate 1) the prevalence of perceived cognitive impairments (PCIs) in Japanese BCSs (hereafter called "survivors") who were treated with chemotherapy, 2) the recognition of these PCIs by other people, and 3) the relationship between PCIs and depression.

\section{Patients and methods}

\section{Study design}

This cross-sectional survey was administered as an anonymous web-based questionnaire to collect demographic information, depressive symptoms, and PCIs related to chemotherapy.

\section{Participants}

We sent three e-mails to BCSs who had registered for a breast cancer advocacy group (BC network), asking them to participate in a web-based survey. The study period was March 1-24, 2014. A sheet explaining the aims and methods of the survey was included with the questionnaire, and return of the completed questionnaire was regarded as consent to participate in the study.

\section{Questionnaire for cognitive impairment}

Previous reports ${ }^{1-3,5,16}$ demonstrated that several domains of cognitive function are affected by chemotherapy. Most studies assessed cognitive function using detailed neurocognitive tests; however, it takes a considerable amount of time to complete those tests. To ensure the test used in our study was both feasible and affordable, we referred to previous reports and drafted a simple questionnaire to evaluate each domain of cognitive function, including memory, concentration, attention, and learning and processing. This questionnaire consisted of an evaluation of PCIs and recognition of PCIs by other people, similar to the Functional Assessment of Cancer Therapy-Cognitive (FACT-Cog, version three) (Figure 1). ${ }^{19}$

i. Have you had any of the following problems since receiving chemotherapy? (more than one answer is allowed)

ii. Have you had any of the following comments from other people since receiving chemotherapy? (More than one answer is allowed)

1. Difficulty concentrating yes/no

2. Slower processing speed in job

or household chores yes/no

3. Shorter attention span yes/no

4. Memory loss yes/no

5. Confusion yes/no

6. Getting lost yes/no

7. Not applicable yes

Figure I Questionnaire to access cognitive impairments.

\section{Questionnaire for psychological condition}

To evaluate psychological status, we used the Japanese version of the Hospital Anxiety and Depression Scale (HADS). ${ }^{21,22}$ Kugaya et al. ${ }^{22}$ reported an optimal cut-off point of 10/11 when screening for adjustment disorder and major depressive disorder in cancer patients, which provided high enough sensitivity and specificity $(91.5 \%$ and $65.4 \%$, respectively) ${ }^{22}$ to determine when to intervene due to psychological distress. Using this cut-off point, we identified the participants who needed intervention for psychological distress (11 HADS score) and categorized them into the depressive group.

\section{Statistical analysis}

Descriptive statistics were used to report sample characteristics, the prevalence of PCIs, and depressive state. The differences among these variables were analyzed by chi-square tests. To analyze the relationship between PCIs and depressive state, a univariate analysis or Student's $t$-test was used, followed by multiple regression analysis. All analyses were performed using the Statistical Package for the Social Sciences (SPSS), version 21.

\section{Results}

The data of $173 \mathrm{BCSs}$ were analyzed. The mean age of the survivors was $50.8 \pm 8.3$ years (mean \pm standard deviation [SD]; range, 34-79 years). Survivors consisted of 170 females (98\%) and three males (2\%). Of the 173 survivors, $98(56.6 \%)$ were treated with chemotherapy. The mean age of the survivors treated with chemotherapy was $50.5 \pm 8.4$ years (range, 34-79 years). They consisted of 96 females $(98 \%)$ and two males $(2 \%)$. The mean time after diagnosis was $0.6 \pm 3.8$ years (range, $0.6-18.5$ years). The types of therapies received were 1) chemotherapy $(\mathrm{n}=98,100 \%), 2)$ hormone therapy $(\mathrm{n}=69$, $70.4 \%), 3)$ radiation therapy $(\mathrm{n}=60,61.2 \%)$, and 4$)$ surgery $(\mathrm{n}=92$, 93.9\%). The mean HADS score was $11.45 \pm 8.08$ (range, 1-33), and 42 $(42.9 \%)$ survivors treated with chemotherapy had HADS scores $\geq 11$. Following the analysis of age, sex ratio, and HADS score, only the HADS score was significantly different between the survivors treated with chemotherapy and the survivors treated without chemotherapy ( $p=0.625,0.724$, and 0.043 , respectively). Detailed demographic data are shown in Table 1.

The prevalence of PCIs was significantly higher among survivors treated with chemotherapy than survivors treated without chemotherapy, except for the item "getting lost" (Table 2). To evaluate the influence of depression on PCIs, we compared the number of PCIs for survivors treated with chemotherapy $(n=56)$ with that of survivors treated without chemotherapy $(\mathrm{n}=28)$ among participants with HADS scores $<11$. Survivors treated with chemotherapy had significantly more cognitive impairments than survivors treated without chemotherapy $(1.77 \pm 1.65$ and $0.89 \pm 1.52$, respectively, $t=2.347, d f=82, p=0.021$ ).

\section{Prevalence of PCIs in survivors treated with chemotherapy and its recognition by other people}

We further analyzed the results of questions about PCIs obtained from the 98 survivors treated with chemotherapy, except for the item "getting lost." The prevalence of having at least one PCI was 77 (79\%) and $42(43 \%)$ survivors treated with chemotherapy had more than three types of PCIs (Figure 2). The prevalence of specific PCIs was as follows: "difficulty concentrating," $46 \%$; "slower processing speed," 56\%; "shorter attention span," 39\%; "memory loss," 44\%; and "confusion," $38 \%$. On the other hand, the prevalence of these PCIs being recognized by other people was significantly lower $(6 \%, 14 \%$, $13 \%, 19 \%$, and $10 \%$, respectively, $p<0.0001$ for all comparisons).

\section{Relationship between number of PCIs and total HADS score}

Because we did not ask about the severity of the PCIs, we treated the number of PCIs as a factor that might adversely influence daily life in further analysis. Multiple regression analysis was performed using a forward stepwise approach, in which variables that were significantly 
related to the total HADS score in the univariate analysis were included according to the strength of the relationship. The analysis showed that the number of PCIs was independently correlated with the total HADS score $(\beta=0.32, p=0.001)$.

Table I demographic characteristic of participants

\begin{tabular}{|c|c|c|c|c|}
\hline \multicolumn{3}{|c|}{ Survivors who treated with chemotherapy $(n=98)$} & \multicolumn{2}{|c|}{$\begin{array}{l}\text { Survivors who treated without chemotherapy } \\
(n=75)\end{array}$} \\
\hline & $n$ & $\%$ & $n$ & $\%$ \\
\hline Age $($ mean $\pm S D)$ & $50.5 \pm 8.4$ & & $51.3 \pm 7.9$ & \\
\hline Female / Male & $96 / 2$ & $98 / 2$ & $74 / 1$ & $98.7 / 1.3$ \\
\hline Period after diagnosis (year) (mean $\pm S D$ ) & $0.6 \pm 3.8$ & & $4.7 \pm 8.7$ & \\
\hline \multicolumn{5}{|l|}{ Type of therapy } \\
\hline Chemotherapy & 98 & 100 & 0 & 0 \\
\hline Hormonal therapy & 69 & 70.4 & 54 & 72 \\
\hline Radiation therapy & 60 & 61.2 & 38 & 50.7 \\
\hline Surgery & 92 & 93.9 & 74 & 98.7 \\
\hline \multicolumn{5}{|l|}{ HADS } \\
\hline$<11$ & 56 & 57.1 & 30 & 40 \\
\hline$\geq 11$ & 42 & 42.9 & 45 & 60 \\
\hline
\end{tabular}

HADS, Hospital Anxiety and Depression Scale; SD, Standard deviation

Table 2 Prevalence of $\mathrm{PCls}$ in survivors $(n=173)$

\begin{tabular}{|c|c|c|c|c|c|}
\hline & & \multicolumn{2}{|c|}{ Experience of chemotherapy } & \multirow{2}{*}{$X^{2}$} & \multirow[b]{2}{*}{$p$ value } \\
\hline & & No & Yes & & \\
\hline \multirow{2}{*}{ Difficulty in concentrating } & No & $53(31.4 \%)$ & $53(31.4 \%)$ & \multirow{2}{*}{7.4} & \multirow{2}{*}{0.006} \\
\hline & Yes & $18(10.7 \%)$ & 45 (26.6\%) & & \\
\hline \multirow{2}{*}{$\begin{array}{l}\text { Slower processing speed in job or } \\
\text { household chores }\end{array}$} & No & $56(32.4 \%)$ & 43 (24.9\%) & \multirow{2}{*}{16.5} & \multirow{2}{*}{$<0.001$} \\
\hline & Yes & 19 (11\%) & 55 (3I.8\%) & & \\
\hline \multirow{2}{*}{ Shorter attention span } & No & $59(34.1 \%)$ & $60(34.7 \%)$ & \multirow{2}{*}{6} & \multirow{2}{*}{0.014} \\
\hline & Yes & $16(9.2 \%)$ & $38(22 \%)$ & & \\
\hline \multirow{2}{*}{ Memory loss } & No & 54 (3I.2\%) & 55 (3I.8\%) & \multirow{2}{*}{4.6} & \multirow{2}{*}{0.032} \\
\hline & Yes & $21(12.1 \%)$ & 43 (24.9\%) & & \\
\hline \multirow{2}{*}{ Confusion } & No & $58(33.5 \%)$ & 61 (35.3\%) & \multirow{2}{*}{4.5} & \multirow{2}{*}{0.034} \\
\hline & Yes & $17(9.8 \%)$ & 37 (2I.4\%) & & \\
\hline \multirow{2}{*}{ Getting lost } & No & $74(42.8 \%)$ & 92 (53.2\%) & \multirow{2}{*}{2.5} & \multirow{2}{*}{0.113} \\
\hline & Yes & I (0.6\%) & $6(3.5 \%)$ & & \\
\hline
\end{tabular}

\section{PCls, Perceived cognitive impairments}

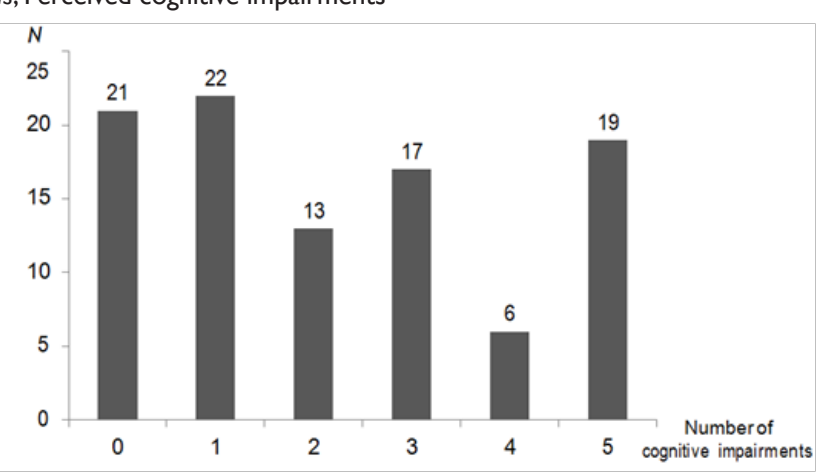

Figure 2 The number of $\mathrm{PCl}$ in survivors treated with chemotherapy ( $n=98)$. PCls, Pereceived cognitive impairments

\section{Discussion}

To the best of our knowledge, this is the first study to investigate the prevalence of PCIs in Japanese BCSs treated with chemotherapy and the recognition of these PCIs by other people. First, we showed that the prevalence of PCIs in six types of cognitive function was significantly higher in survivors treated with chemotherapy than in survivors treated without chemotherapy, except for the item "getting lost" (Table 2) Furthermore, 79\% of survivors treated with chemotherapy perceived cognitive impairments, and $43 \%$ of survivors treated with chemotherapy had more than three types of PCIs (Figure 2). This prevalence is higher than that reported by previous studies that objectively evaluated cognitive impairments by neurocognitive testing. Several studies reported no relationship or a lower relationship between the results of objective neurocognitive tests and 
the subjective perception of cognitive impairments..$^{1,23-25}$ Furthermore, it is often difficult to translate the results of neurocognitive tests to specific problems that BCSs experience in their daily lives. These days, patient-reported outcome data are regarded as more valuable and accurate than objective data, such as clinician-rated data, especially in terms of assessing patients' symptoms and health-related quality of life. ${ }^{26-28}$ Thus, we chose six questions to evaluate each cognitive domain based on previous reports and used those six questions to evaluate the survivors' PCIs. A similarly high PCI prevalence was reported by $\mathrm{Ah}$ et al. ${ }^{18}$ They demonstrated that $94 \%$ of survivors reported clinically significant PCIs, as assessed by FACT-Cog. ${ }^{29}$ These findings, taken together with our results, suggest that PCIs are very common in BCSs, and the questionnaire used in our study may be useful to detect PCIs, especially in a busy clinical setting; however, further validation is required.

Several reports have suggested that PCIs in cancer survivors are affected by mood. Shilling et al. ${ }^{30}$ reported that self-reported cognitive complaints are associated with anxiety, depression, and fatigue. ${ }^{29,30}$ We also found that the prevalence of PCIs was significantly higher in the depressed survivors than in the non-depressed survivors. However, when we analyzed only the data from non-depressed survivors (HADS $<11$ ), the number of PCIs was significantly higher in survivors treated with chemotherapy than in survivors treated without chemotherapy. This result suggests the possibility that some PCIs are independently affected not by mood but by chemotherapy.

Next, we demonstrated a discrepancy between the prevalence of cognitive impairments perceived by survivors treated with chemotherapy and the recognition of these cognitive impairments by other people. The prevalence of cognitive impairments recognized by other people was significantly lower than that of cognitive impairments perceived by survivors treated with chemotherapy. Several previous studies investigated PCIs in BCSs who were undergoing or had undergone chemotherapy using FACT-Cog version three, which consists of four subscales. One subscale assesses PCI (18 items) and another assesses comments from others (4 items). ${ }^{19}$ Both subscale are evaluated the frequency of each occurrence over the 7 days preceding the test on a 5-point Likert-type scale ranging from 0 (never) to 4 (several times per day), indicating that score ranging from 0 to 72 in PCI and 0-16 in comments from others. In the three studies using FACT-Cog, the mean PCI scores and comments from others were 51-63 out of 72 and $15-16$ out of 16 , respectively. ${ }^{31-33}$ Although these results are valuable to our understanding of the prevalence of PCI and the recognition of $\mathrm{PCI}$ by other people, it is difficult to directly compare these factors because the portion of the questionnaire dedicated to PCI and its recognition by other people was different. In the present study, we compared the prevalence of PCI and its recognition by other people using the same questionnaire. To our knowledge, there are no previous reports that compare the prevalence of PCI and its recognition by other people using the same questionnaire. Accordingly, our results should help to understand the discrepancy in the prevalence of PCIs and its recognition by other people in BCSs that underwent chemotherapy. Interestingly, the apparent trend in the prevalence of PCI and its recognition by other people differs from the results of dementia studies. For example, Arlt et al ${ }^{34}$ reported that patients with mild to moderate dementia underestimated their cognitive function, as assessed by self-rating tools, and the estimated cognitive function did not correlate with the Mini-Mental State Examination (MMSE) score. In contrast, the caregivers' assessment of the dementia patients' cognitive functions corresponded with the MMSE scores. ${ }^{34}$ This discrepancy may be due to a lack of insight on the part of the dementia patients. On the other hand, survivors in our study who did not have symptomatic brain metastasis did not lose insight into their physical and mental activity, including cognitive impairments. Although other people, such as family members, usually pay considerable attention to the patients' physical condition, life changes, and prognosis, they likely pay less attention to cognitive changes. Furthermore, cognitive functions affected by chemotherapy appear less impaired to outsiders. Our results may reflect these problems.

Finally, we demonstrated a relationship between PCIs and depression.Because the severity of the depressive state depended on the number of PCIs, having an increased number of cognitive impairments should be considered a risk factor for depression. Similar to our results, a previous study demonstrated that chronic illness, subjective health status, and cognitive impairments were significantly associated with depression among elderly Koreans. ${ }^{35}$ Taken together, these results suggest that the presence of PCI independently affects survivors' moods. Another possibility is that the lack of awareness, concern, and consideration exhibited by other people, especially family members, for day-to-day problems caused by cognitive impairments exacerbates the survivor's psychological distress, leading to depression. Recently, one study reported that PCI and a high HADS score were associated with increased prevalence of selfperceived burden (SPB) on primary caregivers in older patients with hematologic malignancies. ${ }^{36}$ Although the cancer type and mean age were different from those in our study, the coexistence of PCI and depression might also elicit a SPB on family members in BCSs. The limitations of this study were as follows:

a. This was a cross-sectional survey using a simple, clinical self-reported questionnaire to assess cognitive impairments associated with chemotherapy. Therefore, the study design may have created sampling bias, which limits the generalizability of the results. In addition, a variety of detailed questionnaires and other methods for evaluating cognitive impairments have been used in previous studies. Thus, directly comparing the prevalence of cognitive impairments in our data with those of other epidemiological studies is not feasible.

b. The questionnaire we used to assess PCIs was not validated, although it consisted of questions selected from several prior reports and accounted for test feasibility and affordability.

c. We did not ask survivors details about their prescription medications. Thus, we could not rule out other factors influencing PCIs, such as psychotropic agents. In addition, we did not ask for details about their chemotherapy regimens. Thus, we cannot discuss which chemotherapeutic agents may influence cognition.

d. We did not evaluate the severity and frequency of each PCI. Thus, we could not assess the relationships between those factors and depression.

e. To overcome these limitations, prospective large-scale research studies are needed.

\section{Conclusion}

In conclusion, PCIs following chemotherapy are not negligible in Japanese BCSs and could be important factors contributing to the 
development of depression. Oncologists and medical staff should pay attention not only to the survivor's physical condition and psychological distress but also to PCIs. Awareness of PCIs in BCSs may prevent depression or reduce its prevalence.

\section{Funding}

This study was funded by Japanese Medical Support Network (JAMSNET) New York.

\section{Ethics}

Ethical and scientific validity were confirmed by the Institutional Review Board and the Ethics Committee of Osaka University Hospital. This study was conducted in accordance with the Helsinki Declaration.

\section{Acknowledgments}

We would like to thank to Ms. Makiko Yamamoto, a founder of the Young Japanese Breast Cancer Network, the breast cancer patient advocacy group, and the Japanese Medical Support Network (JAMSNET) for supporting our survey research.

\section{Conflict of interest}

The authors declare that they have no conflicts of interest.

\section{References}

1. van Dam FS, Schagen SB, Muller MJ, et al. Impairment of cognitive function in women receiving adjuvant treatment for high-risk breast cancer: high-dose versus standard-dose chemotherapy. J Natl Cancer Inst. 1998;90(3):210-218.

2. Wieneke MH DD. Neuropsychological assessment of cognitive functioning following chemotherapy for breast cancer. Psycooncology. 1995;4(1):61-66.

3. Schagen SB, van Dam FS, Muller MJ, et al. Cognitive deficits after postoperative adjuvant chemotherapy for breast carcinoma. Cancer. 1999;85(3):640-650.

4. Brezden CB, Phillips KA, Abdolell M, et al. Cognitive function in breast cancer patients receiving adjuvant chemotherapy. J Clin Oncol. 2000;18(14):2695-2701.

5. Ahles TA, Saykin AJ, Furstenberg CT, et al. Neuropsychologic impact of standard-dose systemic chemotherapy in long-term survivors of breast cancer and lymphoma. J Clin Oncol. 2002;20(2):485-493.

6. Tchen N, Juffs HG, Downie FP, et al. Cognitive function, fatigue, and menopausal symptoms in women receiving adjuvant chemotherapy for breast cancer. J Clin Oncol. 2003;21(22):4175-4183.

7. Monje M, Dietrich J. Cognitive side effects of cancer therapy demonstrate a functional role for adult neurogenesis. Behav Brain Res. 2012;227(2):376-379.

8. Aluise CD, Miriyala S, Noel T, et al. 2-Mercaptoethane sulfonate prevents doxorubicin-induced plasma protein oxidation and TNFalpha release: implications for the reactive oxygen species-mediated mechanisms of chemobrain. Free Radic Biol Med. 2011;50(11):1630168.

9. Myers JS. The possible role of cytokines in chemotherapy-induced cognitive deficits. Adv Exp Med Biol. 2010;678:119-123.

10. Stemmer SM, Stears JC, Burton BS, et al. White matter changes in patients with breast cancer treated with high-dose chemotherapy and autologous bone marrow support. Am J Neuroradiol. 1994;15(7):12671273.

11. Deprez S, Amant F, Yigit R, et al. Chemotherapy-induced structural changes in cerebral white matter and its correlation with impaired cognitive functioning in breast cancer patients. Hum Brain Mapp. 2011;32(3):480-493.

12. Deprez S, Amant F, Smeets A, et al. Longitudinal assessment of chemotherapy-induced structural changes in cerebral white matter and its correlation with impaired cognitive functioning. J Clin Oncol. 2012;30(3):274-481.

13. Silverman DH, Dy CJ, Castellon SA, et al. Altered frontocortical, cerebellar, and basal ganglia activity in adjuvant-treated breast cancer survivors 5-10 years after chemotherapy. Breast Cancer Res Treat. 2007;103(3):303-311.

14. Argyriou AA, Assimakopoulos K, Iconomou G, et al. Either called "chemobrain" or "chemofog," the long-term chemotherapy-induced cognitive decline in cancer survivors is real. J Pain Symptom Manage. 2011;41(1):126-139.

15. Richardson A, Addington-Hall J, Amir Z, et al. Knowledge, ignorance and priorities for research in key areas of cancer survivorship: findings from a scoping review. British journal of cancer. 2011;105 Suppl 1:S82-S94.

16. Meyers CA, Byrne KS, Komaki R. Cognitive deficits in patients with small cell lung cancer before and after chemotherapy. Lung cancer. 1995;12(3):231-5.

17. Wefel JS, Lenzi R, Theriault RL, et al. The cognitive sequelae of standard-dose adjuvant chemotherapy in women with breast carcinoma: results of a prospective, randomized, longitudinal trial. Cancer. 2004;100(11):2292-2299.

18. Ahles TA, Root JC, Ryan EL. Cancer-and cancer treatment-associated cognitive change: an update on the state of the science. J Clin Oncol. 2012;30(30):3675-86.

19. Wagner L SJ, Butt Z, Lai J, et al. Measuring patient self-reported cognitive function: development of the functional assessment of cancer therapy-cognitive function instrument. J Support Oncol. 2009; 7:W32W39.

20. Zigmond AS, Snaith RP. The hospital anxiety and depression scale. Acta psychiatrica Scandinavica. 1983;67(6):361-70.

21. T K. Hospital Anxiety and Depression Scale. Seisinka Sindangaku. 1993;4:371-372.

22. Kugaya A AT, Okuyama T, Okamura $\mathrm{H}$, et al. Screening for Psychological Distress in Japanese Cancer Patients. Jpn J Clin Oncol. 1998;28(5):333-338.

23. Schagen SB, Muller MJ, Boogerd W, et al. Late effects of adjuvant chemotherapy on cognitive function: a follow-up study in breast cancer patients. Ann Oncol. 2002;13(9):1387-97.

24. Vardy J, Wong K, Yi QL, et al. Assessing cognitive function in cancer patients. Support Care Cancer. 2006;14(11):1111-1118.

25. Ahles TA, Saykin AJ. Breast cancer chemotherapy-related cognitive dysfunction. Clin Breast Cancer. 2002;3 Suppl 3:S84-S90.

26. Administration USDoHaHSFaD. Guidance for Industry PatientReported Outcome Measures: Use in Medical Product Development to Support Labeling Claims. 2009.

27. Velikova G, Wright P, Smith AB, et al. Self-reported quality of life of individual cancer patients: concordance of results with disease course and medical records. J Clin Oncol. 2001;19(7):2064-2073. 
28. Fromme EK, Eilers KM, Mori M, et al. How accurate is clinician reporting of chemotherapy adverse effects? A comparison with patientreported symptoms from the Quality-of-Life Questionnaire C30. J Clin Oncol. 2004;22(17):3485-3490

29. Von Ah D, Tallman EF. Perceived cognitive function in breast cancer survivors: evaluating relationships with objective cognitive performance and other symptoms using the functional assessment of cancer therapy-cognitive function instrument. J Pain Symptom Manage. 2015;49(4):697-706.

30. Shilling V, Jenkins V. Self-reported cognitive problems in women receiving adjuvant therapy for breast cancer. Eur J Oncol Nurs. 2007;11(1):6-15.

31. Cheung YT, Shwe M, Chui WK, et al. Effects of chemotherapy and psychosocial distress on perceived cognitive disturbances in Asian breast cancer patients. Ann Pharmacother. 2012;46(12):1645-1655.

32. Park JH, Bae SH, Jung YS, et al. The psychometric properties of the Korean version of the functional assessment of cancer therapy- cognitive (FACT-Cog) in Korean patients with breast cancer. Support Care Cancer. 2015;23(9):2695-2703.

33. Leach HJ, Danyluk JM, Nishimura KC, et al. Benefits of 24 versus 12 weeks of exercise and wellness programming for women undergoing treatment for breast cancer. Support Care Cancer. 2016;24(11):4597606.

34. Arlt S, Hornung J, Eichenlaub M, et al. The patient with dementia, the caregiver and the doctor: cognition, depression and quality of life from three perspectives. International journal of geriatric psychiatry. 2008;23(6):604-610

35. Park JI, Park TW, Yang JC, et al. Factors associated with depression among elderly Koreans: the role of chronic illness, subjective health status, and cognitive impairment. Psychogeriatrics. 2016;16(1):62-69.

36. Libert $\mathrm{Y}$, Borghgraef $\mathrm{C}$, Beguin $\mathrm{Y}$, et al. Factors associated with self-perceived burden to the primary caregiver in older patients with hematologic malignancies: an exploratory study. Psychooncology. 2016; 26(1):118-124. 